\title{
Effect of Shadowing on the Performance of Space-Time Trellis-Coded Systems
}

\author{
Murat Uysal, Member, IEEE, and Costas N. Georghiades, Fellow, IEEE
}

\begin{abstract}
We analyze the performance of space-time trellis codes over shadowed Rician fading channels. The shadowed Rician channel is a generalization of the Rician model, where the line-of-sight path is subjected to a lognormal transformation due to foliage attenuation, also referred to as shadowing. Using the moment generating function method, we derive an exact expression for the pairwise error probability (PEP) of space-time trellis coded systems operating over this channel. The asymptotic analysis of PEP shows that the design criteria of space-time trellis codes proposed for Rayleigh fading still hold when used over shadowed Rician channels. We also present simulation results for bit-error rate performance under various degrees of shadowing.
\end{abstract}

Index Terms-Pairwise error probability (PEP), Rician fading channels, shadowing, space-time trellis coding.

\section{INTRODUCTION}

$\mathbf{S}$ PACE-TIME trellis coding [1] has been proposed as an effective approach to support high data-rate transmission over fading channels. This technique integrates channel coding, modulation, and multiple transmit antennas with optional receive diversity and is able to exploit the full diversity promised by the multiple-input multiple-output (MIMO) channels. Since its introduction, a significant amount of work has been published on the design, decoding, and applications of this new family of codes (see, for example, [2]-[5]). Most of these results, however, are based on the assumption that the channel fading is either Rayleigh or Rician distributed. The Rician fading channel, which is generally used to model mobile satellite channels, mathematically describes a received signal characterized by a direct (i.e., line-of-sight) component with constant amplitude and a scattered (i.e., multipath) component. The shadowed Rician channel [6] is a generalization of the Rician model, where the line-of-sight path (LOS) is subjected to a lognormal transformation due to foliage attenuation or blockage, also referred to as shadowing. In this paper, we investigate the effects of shadowing on the performance of space-time trellis codes. To the best of our knowledge, no such study exists in the literature.

In their pioneering work on space-time codes, Tarokh et al. [1] provide an upper bound on the pairwise error probability (PEP) using the classical Chernoff bound over Rayleigh and Rician fading channels. In [7], Uysal and Georghiades obtained an

Manuscript received October 8, 2002; revised February 14, 2003; accepted March 19, 2003. The editor coordinating the review of this paper and approving it for publication is $\mathrm{H}$. Jafarkhani. This letter was presented in part at the IEEE Vehicular Technology Conference (VTC-Fall'02), Vancouver, BC, Canada, October 2002.

M. Uysal is with the Department of Electrical and Computer Engineering, University of Waterloo, Waterloo, ON N2L 3G1, Canada (e-mail: muysal@ ece.uwaterloo.ca).

C. N. Georghiades is with the Department of Electrical Engineering, Texas A\&M University, College Station, TX 77843-3128 USA (e-mail: georghia@ee tamu.edu).

Digital Object Identifier 10.1109/TWC.2004.830847 exact expression for PEP, applying the characteristic function technique previously used in the performance analysis of trelliscoded modulation (TCM) to the multiple-antenna transmission problem. Another numerical solution for the same problem is presented by Taricco and Biglieri in [8] using the Gauss-Chebyshev numerical quadrature rule. In [9], Simon presents a simpler method for exactly evaluating the PEP for space-time coded systems based on the moment generating function method. This method allows the evaluation of PEP in a simple closed form and has been previously applied to numerous problems related to the analysis of digital communication systems over fading channels with a single transmit antenna [10]. In this paper, we generalize Simon's work for Rayleigh fading channels in [9] to shadowed Rician fading channels in order to investigate the effects of shadowing on the performance of space-time trellis-coded systems.

\section{SySTEM MODEL}

We consider a wireless communication scenario where the base station is equipped with $M$ transmit antennas and the mobile unit is equipped with $N$ receive antennas. The binary data stream is first modulated and mapped to a sequence of complex modulation symbols. The modulated sequence is then fed to the space-time encoder and transmitted over the wireless channel. The receiver employs a maximum-likelihood decoder and has perfect channel state information (CSI). The received signal in the time interval $l$ at the $n$th receive antenna is given as

$$
r_{l}^{n}=\sum_{m=1}^{M} \alpha_{m, l}^{n} x_{m, l}+\eta_{l}^{n}
$$

where $x_{m, l}$ is a complex-valued modulation symbol transmitted from the $m$ th $(m=1,2, \ldots, M)$ transmit antenna at time $l$, and $\eta_{l}^{n}$ is the additive thermal noise term, the collection of which is modeled as independent samples of a zero-mean complex Gaussian random variable with variance $N_{0} / 2$ per dimension. The fading coefficient $\alpha_{m, l}^{n}$ represents the channel gain from transmit antenna $m$ to receive antenna $n$ in time interval $l$. In our case, the fading amplitude is described by a shadowed Rician fading model, with Rician and Rayleigh channels being special cases. The $\alpha_{m, l}^{n}$ 's are modeled as independent and identically distributed (i.i.d) samples of complex Gaussian random variables with mean $E\left(\alpha_{m, l}^{n}\right)$ and variance $\operatorname{var}\left(\alpha_{m, l}^{n}\right)=\sigma_{\alpha}^{2}$ per dimension. In this model, the LOS (corresponding to the nonzero mean) component is not constant but rather a lognormally distributed random variable.

\section{Derivation of PAIRWISE ERror Probability}

In this section, we derive an exact expression for the PEP for two different cases: 1) the quasi-static channel and 2) the 
symbol-by-symbol perfectly interleaved channel (which is referred to as "fast fading" in [1]).

\section{A. Quasi-Static Fading Channel}

In the quasi-static channel, the fading coefficients remain constant over the duration of one frame. Therefore, $\alpha_{m, l}^{n}=\alpha_{m}^{n}$ for $l=1,2, \ldots, L$, where $L$ is the number of symbols in a frame. Let $\boldsymbol{x}_{l}=\left(x_{1, l}, x_{2, l}, \ldots, x_{M, l}\right)$ denote the code vector transmitted from the $M$ transmit antennas in time interval $l$. For a frame length of $L$ time intervals, we denote the codeword matrix as $\boldsymbol{X}=\left[\boldsymbol{x}_{1}^{T}, \boldsymbol{x}_{2}^{T}, \ldots, \boldsymbol{x}_{L}^{T}\right]^{1}$. The PEP represents the probability of choosing the code matrix $\hat{X}$ when indeed $\boldsymbol{X}$ was transmitted. Under the assumption of perfect CSI, the conditional PEP is given by

$$
\begin{aligned}
P\left(\boldsymbol{X}, \hat{\boldsymbol{X}} \mid \alpha_{m}^{n}, m=1, \ldots M,\right. & n=1, \ldots N) \\
& =Q\left(\sqrt{\frac{E_{s}}{2 N_{0}} d^{2}(\boldsymbol{X}, \hat{\boldsymbol{X}})}\right)
\end{aligned}
$$

where $Q(\cdot)$ is the Gaussian $Q$ function, $E_{s}$ is the energy per symbol, and $d^{2}(\boldsymbol{X}, \hat{\boldsymbol{X}})$ is the squared Euclidean distance between the transmitted and decoded code sequences, expressed as

$$
d^{2}(\boldsymbol{X}, \hat{\boldsymbol{X}})=\sum_{n=1}^{N} \sum_{l=1}^{L}\left|\sum_{m=1}^{M} \alpha_{m}^{n}\left(x_{m, l}-\hat{x}_{m, l}\right)\right|^{2} .
$$

Defining the channel vector $\boldsymbol{\alpha}^{n}=\left(\alpha_{1}^{n}, \alpha_{2}^{n}, \ldots, \alpha_{M}^{n}\right)$ and the codeword difference matrix

$\boldsymbol{X}-\hat{\boldsymbol{X}}=\left[\begin{array}{cccc}x_{1,1}-\hat{x}_{1,1} & x_{1,2}-\hat{x}_{1,2} & \cdots \cdots & x_{1, L}-\hat{x}_{1, L} \\ x_{2,1}-\hat{x}_{2,1} & x_{2,2}-\hat{x}_{2,2} & \cdots \cdots & x_{2, L}-\hat{x}_{2, L} \\ \cdot & \cdot & & \cdot \\ \cdot & \cdot & & \cdot \\ x_{M, 1}-\hat{x}_{M, 1} & x_{M, 2}-\hat{x}_{M, 2} & \cdots \cdots & x_{M, L}-\hat{x}_{M, L}\end{array}\right]$.

Equation (2) can be rewritten as

$$
\begin{aligned}
P\left(\boldsymbol{X}, \hat{\boldsymbol{X}} \mid \alpha_{m}^{n}, m=1, \ldots M, n=1, \ldots N\right) \\
\quad=Q\left(\sqrt{\frac{E_{s}}{2 N_{0}} \sum_{n=1}^{N} \boldsymbol{\alpha}^{n} \boldsymbol{A}\left(\boldsymbol{\alpha}^{n}\right)^{H}}\right)
\end{aligned}
$$

where $\boldsymbol{A}=(\boldsymbol{X}-\hat{\boldsymbol{X}})(\boldsymbol{X}-\hat{\boldsymbol{X}})^{H}$. Since $\boldsymbol{A}$ is Hermitian, there exists a unitary matrix $\boldsymbol{V}$ and a real diagonal matrix $\boldsymbol{D}$ such that $\boldsymbol{V} \boldsymbol{A} \boldsymbol{V}^{H}=\boldsymbol{D}$. The columns of $\boldsymbol{V}$, i.e., $\boldsymbol{v}_{k}=\left(v_{k, 1}, v_{k, 2}, \ldots, v_{k, M}\right)^{T}, k=1,2, \ldots M$ form a complete orthonormal basis given by the eigenvectors of $A$ and the diagonal elements of $\boldsymbol{D}$ are the eigenvalues of $\boldsymbol{A}$. Denoting the (nonnegative) eigenvalues of $\boldsymbol{A}$ by $\lambda_{m}$, we obtain

$$
\boldsymbol{\alpha}^{n} \boldsymbol{A}\left(\boldsymbol{\alpha}^{n}\right)^{H}=\sum_{m=1}^{M} \lambda_{m}\left|\beta_{m}^{n}\right|^{2}, \quad \text { with } \quad \beta_{m}^{n}=\sum_{k=1}^{M} \alpha_{k}^{n} v_{k, m}^{*}
$$

${ }^{1}$ Throughout the paper, we use $(\cdot)^{T}$ and $(\cdot)^{H}$ to represent transpose and conjugate transpose operations, respectively. where $\beta_{m}^{n}$ is the $m$ th element of the $M$-length vector $\beta^{n}=\boldsymbol{\alpha}^{n} V^{H}$. Recalling that the fading coefficients are complex Gaussian random variables, the $\beta_{m}^{n}$ 's are also complex Gaussian with mean and variance

$$
\begin{gathered}
\mu_{m, n}=E\left(\boldsymbol{\alpha}^{n}\right) \boldsymbol{V}^{H}=\sum_{k=1}^{M} E\left(\alpha_{k}^{n}\right) v_{k, m}^{*} \\
\sigma^{2}=\operatorname{var}\left(\alpha_{m}^{n}\right) \sum_{k=1}^{M}\left|v_{k, m}\right|^{2}=\sigma_{\alpha}^{2} .
\end{gathered}
$$

Therefore, $\beta_{m}^{n}$ has the same variance as $\alpha_{m}^{n}$ but a different mean, which is found as a linearly weighted sum of the mean values of $\alpha_{m}^{n}$. Since $\beta_{m}^{n}$ is complex Gaussian, its amplitude $\left|\beta_{m}^{n}\right|$ has a Rician conditional distribution (with respect to $\mu_{m, n}$ ) with probability density function

$$
\begin{aligned}
& p_{\left|\beta_{m}^{n}\right| \mid \mu_{m, n}}\left(\left|\beta_{m}^{n}\right| \mid \mu_{m, n}\right) \\
& \quad=\frac{\left|\beta_{m}^{n}\right|}{\sigma^{2}} \exp \left(-\frac{\left|\beta_{m}^{n}\right|^{2}+\mu_{m, n}^{2}}{2 \sigma^{2}}\right) I_{0}\left(\frac{\left|\beta_{m}^{n}\right| \mu_{m, n}}{\sigma^{2}}\right)
\end{aligned}
$$

where $I_{0}(\cdot)$ is the zero-order modified Bessel function of the first kind; the probability density function of the LOS component (mean of $\beta_{m}^{n}$ ) is given by the lognormal distribution ${ }^{2}$

$$
p_{\mu_{m, n}}\left(\mu_{m, n}\right)=\frac{1}{\sqrt{2 \pi} \sigma_{\mu} \mu_{m, n}} \exp \left(-\frac{\left(\ln \mu_{m, n}-m_{\mu}\right)^{2}}{2 \sigma_{\mu}^{2}}\right) .
$$

The parameters $\sigma, \sigma_{\mu}$, and $m_{\mu}$ in (5) and (6) specify the degree of shadowing.

In order to derive the unconditional PEP from (4), we employ the moment generating function (MGF) technique [10]. It is easy to see that the argument under the square-root sign in (4) is a quadratic form of complex Gaussian random variables. If we define the random variable

$$
\Gamma=\frac{E_{s}}{2 N_{0}} \sum_{n=1}^{N} \boldsymbol{\alpha}^{n} \boldsymbol{A}\left(\boldsymbol{\alpha}^{n}\right)^{H}
$$

its MGF is given in [11] and [12] as

$$
\Phi_{\Gamma}(s)=\prod_{n=1}^{N} \prod_{m=1}^{M} \frac{1}{1-s \chi_{m}} \exp \left(\frac{s \chi_{m}\left|d_{m}\right|^{2}}{1-s \chi_{m}}\right)
$$

where $\chi_{m}$ are the eigenvalues of $\left(E_{s} / 2 N_{0}\right) \boldsymbol{\Sigma} \boldsymbol{A}$ with $\boldsymbol{\Sigma}$ the covariance matrix of $\boldsymbol{\alpha}^{n}$, and $d_{m}$ are the elements of the $M$-length vector $\boldsymbol{d}=\mathrm{E}\left(\boldsymbol{\alpha}^{n}\right) \boldsymbol{V}^{H} \boldsymbol{\Sigma}^{-1 / 2}$. It can be easily shown that, in our case, $\chi_{m}=\left(E_{s} / 2 N_{0}\right) 2 \sigma^{2} \lambda_{m}$ and $\left|d_{m}\right|^{2}=\mu_{m, n}^{2} / 2 \sigma^{2}$. Using the alternative form of the Gaussian $Q$-function [10], the average of the PEP over the channel statistics $\alpha_{m}^{n}$ (while $\mu_{m, n}$ is still treated as a constant) is given by

$$
\begin{aligned}
& P\left(\boldsymbol{X}, \hat{\boldsymbol{X}} \mid \mu_{m, n}, m=1, \ldots M, n=1, \ldots N\right) \\
&=\frac{1}{\pi} \int_{0}^{\pi / 2} \Phi_{\Gamma}\left(-\frac{1}{2 \sin ^{2} \theta}\right) d \theta .
\end{aligned}
$$


Replacing (7) in (8), we obtain

$$
\begin{aligned}
& P\left(\boldsymbol{X}, \hat{\boldsymbol{X}} \mid \mu_{m, n}, m=1, \ldots M, n=1, \ldots N\right) \\
& =\frac{1}{\pi} \int_{0}^{\pi / 2} \prod_{n=1}^{N} \prod_{m=1}^{M} \frac{1}{1+\frac{\Omega_{m}}{\sin ^{2} \theta}} \exp \left(-\frac{\mu_{m, n}^{2}}{2 \sigma^{2}} \frac{\frac{\Omega_{m}}{\sin ^{2} \theta}}{1+\frac{\Omega_{m}}{\sin ^{2} \theta}}\right) d \theta
\end{aligned}
$$

where $\Omega_{m}=\chi_{M} / 2=\left(E_{s} / 4 N_{0}\right) 2 \sigma^{2} \lambda_{m}$. In order to find the unconditional PEP, we still need to take an expectation of (9) with respect to $\mu_{m, n}$, whose distribution is given by (6). Using the i.i.d. properties of $\mu_{m, n}$, this expectation yields

$$
\begin{aligned}
P(\boldsymbol{X}, \hat{\boldsymbol{X}})= & \frac{1}{\pi} \int_{\theta=0}^{\pi / 2} \prod_{m=1}^{M}\left[\frac{1}{1+\frac{\Omega_{m}}{\sin ^{2} \theta}} \frac{1}{\sqrt{2 \pi} \sigma_{\mu}}\right. \\
& \times \int_{\mu_{m, n}=0}^{\infty} \frac{1}{\mu_{m, n}} \exp \left(-\frac{\mu_{m, n}^{2}}{2 \sigma^{2}} \frac{\frac{\Omega_{m}}{\sin ^{2} \theta}}{1+\frac{\Omega_{m}}{\sin ^{2} \theta}}\right) \\
& \left.\times \exp \left(-\frac{\left(\ln \mu_{m, n}-m_{\mu}\right)^{2}}{2 \sigma^{2}}\right) d \mu_{m, n}\right]^{N} d \theta .
\end{aligned}
$$

Introducing the variable change $u_{m, n}=\left(\ln \mu_{m, n}-\right.$ $\left.m_{\mu}\right) / \sqrt{2 \sigma_{\mu}^{2}}$, (10) can be rewritten as

$$
\begin{aligned}
& P(\boldsymbol{X}, \hat{\boldsymbol{X}}) \\
& =\frac{1}{\pi} \int_{\theta=0}^{\pi / 2} \prod_{m=1}^{M}\left[\frac{1}{1+\frac{\Omega_{m}}{\sin ^{2} \theta}} \frac{1}{\sqrt{\pi}} \int_{u_{m, n}=-\infty}^{\infty} \exp \left(-u_{m, n}^{2}\right)\right. \\
& \quad \times \exp \left(-\frac{1}{2 \sigma^{2}} \frac{\frac{\Omega_{m}}{\sin ^{2} \theta}}{1+\frac{\Omega_{m}}{\sin ^{2} \theta}}\right. \\
& \left.\left.\quad \times \exp \left(2 \sqrt{2} \sigma_{\mu} u_{m, n}+2 m_{\mu}\right)\right) d u_{m, n}\right]^{N} d \theta
\end{aligned}
$$

The inner integral has the form of $\int_{-\infty}^{\infty} f(u) \exp \left(-u^{2}\right) d u$. It is possible to show that such an integral can be expressed in terms of an infinite sum (see the Appendix). This yields the final form of exact PEP as

$$
\begin{aligned}
& P(\boldsymbol{X}, \hat{\boldsymbol{X}})=\frac{1}{\pi} \int_{\theta=0}^{\pi / 2} \prod_{m=1}^{M}\left\{\frac{1}{1+\frac{\Omega_{m}}{\sin ^{2} \theta}} \exp \left(-\Delta_{m}(\theta)\right)\right. \\
& \left.\times\left[1+\sum_{\substack{k=2 \\
k \text { even }}}^{\infty} \frac{(k-1) ! !}{k !}\left(2 \sigma_{\mu}\right)^{k} \sum_{t=1}^{k} g_{k, t}\left(\Delta_{m}(\theta)\right)^{t}\right]\right\}^{N} d \theta
\end{aligned}
$$

where

$$
\Delta_{m}(\theta)=\frac{1}{2 \sigma^{2}} \frac{\frac{\Omega_{m}}{\sin ^{2} \theta}}{1+\frac{\Omega_{m}}{\sin ^{2} \theta}} \exp \left(2 m_{\mu}\right)
$$

The coefficients $g_{k, t}$ in (12) can be computed through the recursive equation

$$
g_{k, t}=t g_{k-1, t}-g_{k-1, t-1}, \quad \text { with } g_{k, 1}=-1
$$

for $k=1,2 \ldots$, and $g_{k, t}=0$ for $t>k$ given in the Appendix.

It will be also interesting to point out how (12) relates to the unshadowed case. Assuming there is no shadowing, then the LOS component is no longer a random variable and just given as a constant equal to its mean $\mu_{m, n}=\exp \left(2 m_{\mu}\right)$. For the Rayleigh fading case, $m_{\mu} \rightarrow-\infty$, leading to a zero LOS component, i.e., $\mu_{m, n}=0$. Furthermore, inserting $\sigma_{\mu}^{2}=0$ and $\sigma^{2}=0.5$ in (12), we obtain

$$
P(\boldsymbol{X}, \hat{\boldsymbol{X}})=\frac{1}{\pi} \int_{\theta=0}^{\pi / 2} \prod_{m=1}^{M}\left(1+\frac{E_{s}}{4 N_{0}} \frac{\lambda_{m}}{\sin ^{2} \theta}\right)^{-N} d \theta
$$

which is the PEP expression presented in [9] when the eigenvalues are now given by

$$
\lambda_{m}=\sum_{l=1}^{L}\left|x_{m, l}-\hat{x}_{m, l}\right|^{2} .
$$

for orthogonal error events.

The derivation of PEP for shadowed Rician channels also allows us to examine the design criteria for space-time codes. In [1], Tarokh et al. proposed the rank criterion and determinant criterion for quasi-static Rayleigh fading channels, which determine the diversity and coding gain, respectively. Specifically, from the analysis of PEP for high signal-to-noise ratio (SNR), they prove that a diversity advantage of $r$ and a coding advantage of $\left(\lambda_{1} \lambda_{2} \ldots \lambda_{r}\right)^{1 / r}$ are achieved, where $r$ denotes the rank of matrix $\boldsymbol{A}$. Similar to the analysis presented in [1], setting $\theta=\pi / 2$ in (12) ([10, p. 506]) and assuming asymptotically high SNR, an upper bound on PEP is found as

$$
P(\boldsymbol{X}, \hat{\boldsymbol{X}}) \leq\left(\frac{E_{s}}{4 N_{0}}\right)^{-r N}\left(\prod_{m=1}^{r} \lambda_{m}\right)^{-N}\left[g\left(\sigma, \sigma_{\mu}, m_{\mu}\right)\right]^{r N}
$$

where

$$
\begin{aligned}
g\left(\sigma, \sigma_{\mu}, m_{\mu}\right) & \\
= & \frac{1}{2 \sigma^{2}} \exp \left(-\frac{1}{2 \sigma^{2}} \exp \left(2 m_{\mu}\right)\right) \\
& \times\left[1+\sum_{\substack{k=2 \\
k: \text { even }}}^{\infty} \frac{(k-1) ! !}{k !}\left(2 \sigma_{\mu}\right)^{k}\right. \\
& \left.\times \sum_{t=1}^{k} g_{k, t}\left(\frac{1}{2 \sigma^{2}} \exp \left(2 m_{\mu}\right)\right)^{t}\right] .
\end{aligned}
$$

result is essentially the same as [1, Eq. 10], except for the presence of the factor $\left[g\left(\sigma, \sigma_{\mu}, m_{\mu}\right)\right]$. Therefore, the same diversity advantage (i.e., $r N$ ) is obtained over the shadowed Rician channel as in a Rayleigh channel. The additional factor in (16) is completely characterized by the channel parameters and it can be considered as a coding gain variation due to shadowing. Since the rank criterion is the primary design criterion for space-time 
trellis codes, the first step in "good" code design is maximization of the diversity order. Therefore, once $r$ is optimized, this additional factor becomes just a constant. This allows us to conclude that the rank criterion and determinant criterion [1] still hold for the shadowed Rician channel.

\section{B. Symbol-by-Symbol Interleaved Fading Channel}

Here, we assume that the fading coefficient is constant over one symbol interval and changes from one symbol to another in an independent manner. For the interleaved channel, the squared Euclidean distance between the transmitted and decoded code sequences is given as

$$
d^{2}(\boldsymbol{X}, \hat{\boldsymbol{X}})=\sum_{n=1}^{N} \sum_{l=1}^{L}\left|\sum_{m=1}^{M} \alpha_{m, l}^{n}\left(x_{m, l}-\hat{x}_{m, l}\right)\right|^{2} .
$$

It is different from that for quasi-static channels, given by (3), due to the fact that now the fading coefficient is time-dependent.

Defining the channel vector $\boldsymbol{\alpha}_{l}^{n}=\left(\alpha_{1, l}^{n}, \alpha_{2, l}^{n}, \ldots, \alpha_{M, l}^{n}\right)$ and the code vector $\boldsymbol{x}_{l}=\left\langle x_{1, l}, x_{2, l}, \ldots, x_{M, l}\right\rangle$, the unconditional PEP can be written as

$$
\begin{aligned}
P\left(\boldsymbol{X}, \hat{\boldsymbol{X}} \mid \alpha_{m, l}^{n}, m\right. & =1, \ldots M, n=1, \ldots N, l=1, \ldots L) \\
= & Q\left(\sqrt{\frac{E_{s}}{2 N_{0}} \sum_{n=1}^{N} \sum_{l=1}^{L} \boldsymbol{\alpha}_{l}^{n} \boldsymbol{A}_{l}\left(\boldsymbol{\alpha}_{l}^{n}\right)^{H}}\right)
\end{aligned}
$$

where $\boldsymbol{A}_{\boldsymbol{l}}=\left(\boldsymbol{x}_{\boldsymbol{l}}-\hat{\boldsymbol{x}}_{l}\right)\left(\boldsymbol{x}_{l}-\hat{\boldsymbol{x}}_{l}\right)^{H}$. It is easy to see that the eigenvalues of $A_{l}$ are simply given as

$$
\lambda_{l}=\sum_{m=1}^{M}\left|x_{m, l}-\hat{x}_{m, l}\right|^{2} .
$$

Following along lines similar to those presented in the previous section, we obtain the exact PEP as

$$
\begin{aligned}
& P(\boldsymbol{X}, \hat{\boldsymbol{X}})=\frac{1}{\pi} \int_{\theta=0}^{\pi / 2} \prod_{l=1}^{L}\left\{\frac{1}{1+\frac{\Omega_{l}}{\sin ^{2} \theta}} \exp \left(-\Delta_{l}(\theta)\right)\right. \\
& \left.\times\left[1+\sum_{\substack{k=2 \\
k \text { even }}}^{\infty} \frac{(k-1) ! !}{k !}\left(2 \sigma_{\mu}\right)^{k} \sum_{t=1}^{k} g_{k, t}\left(\Delta_{l}(\theta)\right)^{t}\right]\right\}^{N} d \theta
\end{aligned}
$$

where

and

$$
\Delta_{l}(\theta)=\frac{1}{2 \sigma^{2}} \frac{\Omega_{l} / \sin ^{2} \theta}{1+\Omega_{l} / \sin ^{2} \theta} \exp \left(2 m_{\mu}\right)
$$

$$
\Omega_{l}=\frac{E_{s}}{4 N_{0}} 2 \sigma^{2} \sum_{m=1}^{M}\left|x_{m, l}-\hat{x}_{m, l}\right|^{2} .
$$

It is worth noting that the integral argument in (21) is given as a product over time instances while the PEP for the quasi-static channels is expressed as a product over the number of transmit antennas. Therefore, the use of space-time codes over interleaved channels does not result in spatial diversity, but only achieves an improved time diversity, due to the increase in coding gain. These conclusions are identical to those in [9] for the Rayleigh channel. Furthermore, inserting $\sigma_{\mu}^{2}=0$ and $\sigma^{2}=0.5$ in (21), we obtain

$$
\begin{aligned}
& P(\boldsymbol{X}, \hat{\boldsymbol{X}}) \\
& =\frac{1}{\pi} \int_{\theta=0}^{\pi / 2} \prod_{l=1}^{L}\left(1+\frac{1}{\sin ^{2} \theta} \frac{E_{s}}{4 N_{0}} \sum_{m=1}^{M}\left|x_{m, l}-\hat{x}_{m, l}\right|^{2}\right)^{-N}
\end{aligned}
$$

which is identical to the results in [9] for Rayleigh fading channels.

Now, we examine the asymptotic behavior of the PEP. Let $\varpi$ denote the set of time instances $1 \leq l \leq L$ such that $\boldsymbol{X} \neq \hat{\boldsymbol{X}}$, and let $|\varpi|$ denote the number of elements in $\varpi$, i.e., the cardinality of the set. Then, it follows from (21) that for asymptotically high SNR values

$$
\begin{aligned}
P(\boldsymbol{X}, \hat{\boldsymbol{X}}) \leq\left(\frac{E_{s}}{4 N_{0}}\right)^{-|\varpi| N} & \left(\prod_{l=1}^{|\varpi|} \sum_{m=1}^{M}\left|x_{m, l}-\hat{x}_{m, l}\right|^{2}\right)^{-N} \\
& \times\left[g\left(\sigma, \sigma_{\mu}, m_{\mu}\right)\right]^{|\varpi| N}
\end{aligned}
$$

This result is essentially the same as [1, Eq. 17], except for the presence of the factor $\left[g\left(\sigma, \sigma_{\mu}, m_{\mu}\right)\right]^{|\varpi| N}$. As was true for the quasi-static channel, the shadowing results only in a coding variation due to the presence of an additional factor, but the design criteria originally proposed in [1] still hold.

\section{NUMERICAL RESULTS}

As an example for the shadowed Rician model, we consider the Canadian Mobile Satellite channel [6], [13]. Reference [13, Table I] shows the values of the shadowing parameters for this channel, which are determined by empirical fit to measured data within Canada. In this table, the terms light, average, and heavy are used to represent an increasing effect of the shadowing. We consider the four-state quaternary phase-shift keying (QPSK) space-time trellis code in [1] as an example. This is also the same example considered in [7, Fig. 1] and [9, Fig. 1]. For the shortest error event of this code, the matrix $\boldsymbol{A}=(\boldsymbol{X}-\hat{\boldsymbol{X}})(\boldsymbol{X}-$ $\hat{\boldsymbol{X}})^{H}=4 \boldsymbol{I}$ is diagonal and has two identical eigenvalues equal to 4 . Inserting the eigenvalues and truncating the inner infinite sum in (12) to its first term (i.e., fix $k=2$ ), we obtain

$$
\begin{aligned}
P(\boldsymbol{X}, \hat{\boldsymbol{X}})=\frac{1}{\pi} \int_{\theta=0}^{\pi / 2}\left\{\left(1+\frac{E_{s}}{N_{0}} \frac{2 \sigma^{2}}{\sin ^{2} \theta}\right)^{-1} \exp (-\Delta(\theta))\right. \\
\left.\times\left[1-2 \sigma_{\mu}^{2} \Delta(\theta)+2 \sigma_{\mu}^{2}(\Delta(\theta))^{2}\right]\right\}^{2} d \theta
\end{aligned}
$$

where

$$
\Delta(\theta)=\frac{1}{2 \sigma^{2}} \frac{\left(E_{s} / N_{0}\right)\left(2 \sigma^{2} / \sin ^{2} \theta\right)}{1+\left(E_{s} / N_{0}\right)\left(2 \sigma^{2} / \sin ^{2} \theta\right)} \exp \left(2 m_{\mu}\right) .
$$

It should be noted that using more terms for the infinite sum in (12) does not result in a visible change in the plots for the considered practical values of shadowing. In Fig. 1, we plot the exact PEP results for the different degrees of shadowing. To allow comparison with a similar analysis for trellis coded modulation in the single antenna case [14], we also include PEP results for different values of the Rician parameter $K$ [15]. It is observed 


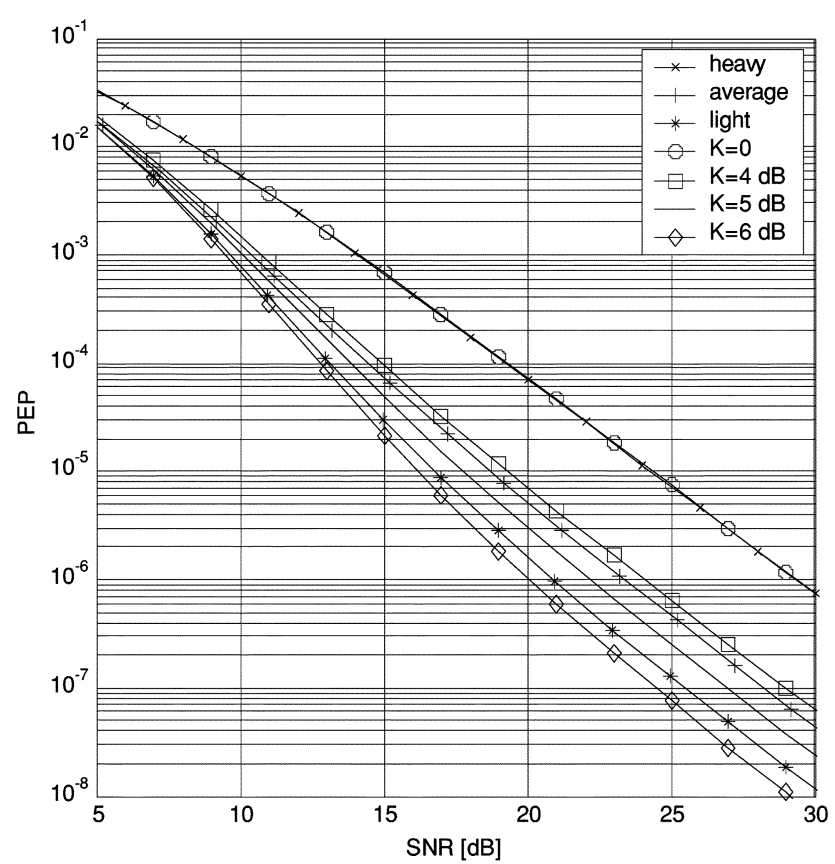

Fig. 1. Pairwise error probability for different degrees of shadowing.

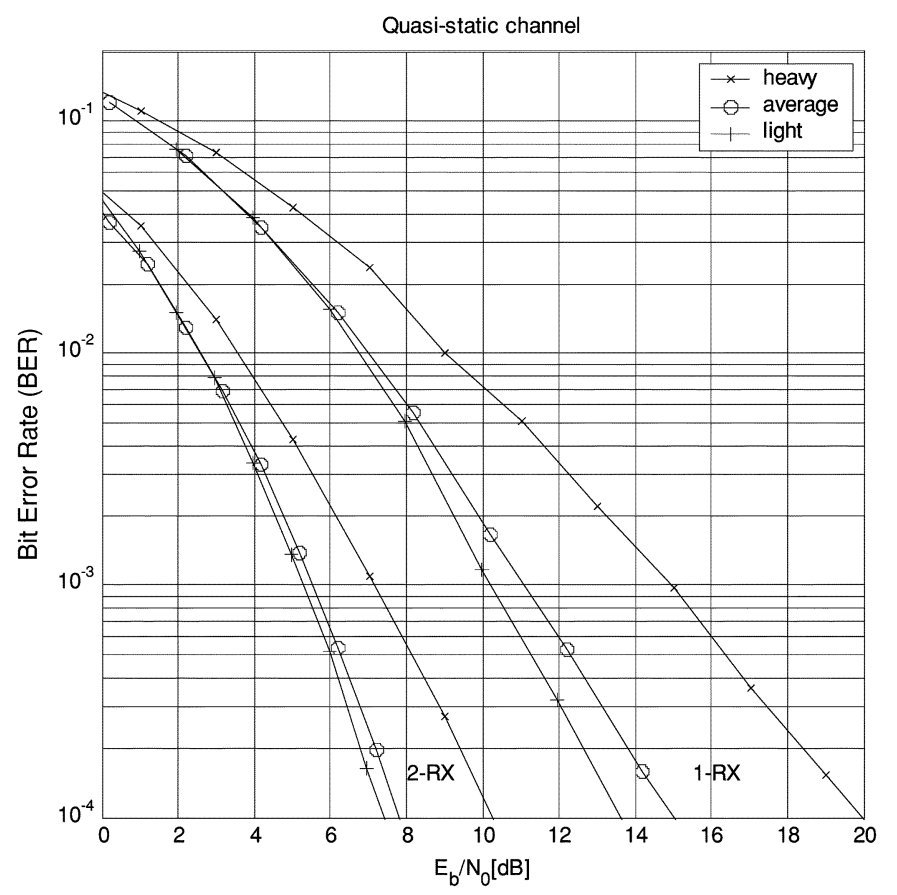

Fig. 2. BER performance for the four-state space-time code over the quasistatic channel.

that the heavy shadowing performance matches that of Rayleigh fading (i.e., $K=0$ ). Average and light shadowing give equivalent performances to Rician fading channels with $K=4-5 \mathrm{~dB}$ and $K=5-6 \mathrm{~dB}$, respectively. These observations agree with the results in [14].

In the following, we focus on the bit-error rate (BER) results obtained through Monte Carlo simulation. BER performance results over quasi-static channels and interleaved channels are illustrated in Figs. 2 and 3, respectively. Over the quasi-static channel, at BER $=10^{-3}$, approximately 1 - and 5-dB degrada-

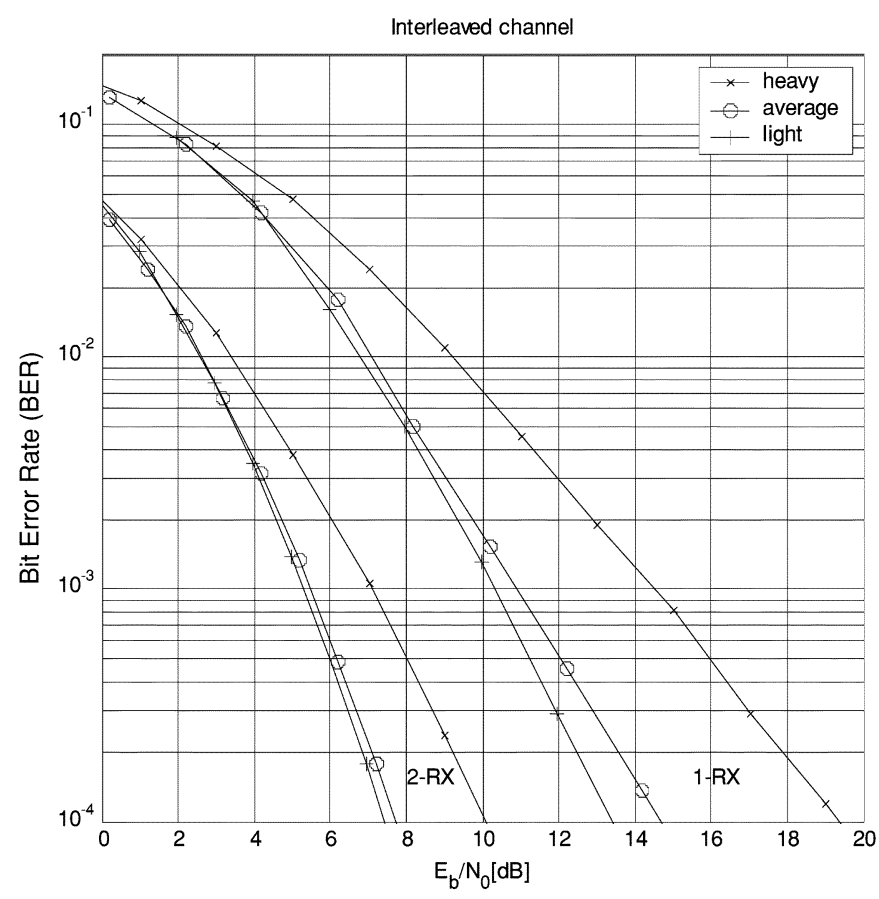

Fig. 3. BER performance for the four-state space-time code over the interleaved channel.

tions are observed for average and heavy shadowing compared to the light shadowing case, respectively. The degradation is less severe for the two receive antenna case since the overall channel looks more like Gaussian with the additional receive diversity. Comparison of Figs. 2 and 3 reveals that the four-state code gives the same diversity order regardless of whether interleaving is used or not. There is only a slight coding gain obtained by the use of interleaving. This can be easily explained based on the code parameters. As shown by (16) for the quasi-static channel, the diversity order is determined by $r N$. Since this code is designed to provide full diversity [1], $r$ is equal to the number of transmit antennas, which is two in this case. On the other hand, for the interleaved channel, the diversity order is given as $|\varpi| N$ based on (24). Since the effective code length (ECL) of this code (i.e., the smallest value of $|\varpi|$ over all possible error events) is 2 , the diversity order is achieved again as 2 , which is the same as for the quasi-static channel. This is a result of the coincidental fact that the ECL is equal to the number of transmit antennas for this specific example.

\section{CONCLUSION}

We investigated the effect of shadowing which is encountered in some mobile satellite channels on the performance of space-time trellis-coded systems. Both quasi-static and interleaved fading channels were considered. The analysis was carried out via the derivation of an exact PEP expression using the MGF technique. Asymptotic PEP analysis reveals that the rank criterion and determinant criterion originally proposed for the Rayleigh fading channel still hold for this case. Monte Carlo simulation results were also presented to demonstrate the BER performance of space-time trellis codes under various degrees of shadowing. 


\section{APPENDIX}

This Appendix evaluates the inner integral in (11) in terms of an infinite sum. Defining

$$
\begin{aligned}
& a=\frac{1}{2 \sigma^{2}} \frac{\Omega_{m} / \sin ^{2} \theta}{1+\Omega_{m} / \sin ^{2} \theta} \\
& b=2 \sqrt{2} \sigma_{\mu}
\end{aligned}
$$

and

$$
c=2 m_{\mu}
$$

the inner integral in (11) can be rewritten as

$$
\int_{-\infty}^{\infty} f(u) \exp \left(-u^{2}\right) d u
$$

with $f(u)=\exp (-a \exp (b u+c))$. Expanding $f(u)$ in a Taylor series, we obtain

$$
\int_{-\infty}^{\infty} f(u) \exp \left(-u^{2}\right) d u=\sum_{k=0}^{\infty} \frac{f^{k}(0)}{k !} \int_{-\infty}^{\infty} u^{k} \exp \left(-u^{2}\right) d u
$$

where $f^{k}(0)$ are the Taylor series coefficients and, in our case, they can be determined as

$$
f^{k}(0)=\exp (-a \exp (c)) b^{k} \sum_{t=1}^{k} g_{k, t}(a \exp (c))^{t}
$$

where $g_{k, t}$ can be computed by the recursive equation

$$
g_{k, t}=t g_{k-1, t}-g_{k-1, t-1} \quad \text { with } \quad g_{k, 1}=-1
$$

for $k=1,2 \ldots$ and $g_{k, t}=0$ for $t>k$. Using the integral form given by ([16], p. 382, (3.462.1)), it can be easily shown that the integral in (29) is zero for odd values of $k$. For even values of $k$, we can use the result ([16, p. 382, (3.461.4)]) and express (29) as

$$
\int_{-\infty}^{\infty} f(u) \exp \left(-u^{2}\right) d u=\sqrt{\pi} \sum_{k=0}^{\infty} \frac{f^{k}(0)}{k !} \frac{(k-1) ! !}{2^{k / 2}} .
$$

Replacing (28) by (31) and using (29) with $a, b$, and $c$ values given as in (26), one can obtain the final form for the inner integral of (11), leading to (12).

\section{ACKNOWLEDGMENT}

The authors would like to thank anonymous reviewers, especially Reviewer 1, for helpful comments to improve the presentation of the paper.

\section{REFERENCES}

[1] V. Tarokh, N. Seshadri, and A. R. Calderbank, "Space-time codes for high data rate wireless communication: Performance criterion and code construction," IEEE Trans. Inform. Theory, vol. 44, pp. 744-765, Mar. 1998.

[2] A. Naguib, V. Tarokh, N. Seshadri, and A. R. Calderbank, "A space-time coding modem for high-data-rate wireless communications," IEEE J. Select. Areas Commun., vol. 16, pp. 1459-1478, Oct. 1998.

[3] V. Tarokh, A. Naguib, N. Seshadri, and A. R. Calderbank, "Space-time codes for high data rate wireless communication: Performance criteria in the presence of channel estimation errors, mobility and multiple paths," IEEE Trans. Commun., vol. 47, pp. 199-207, Feb. 1999.

[4] —_, "Combined array processing and space-time coding," IEEE Trans. Inform. Theory, vol. 45, pp. 1121-1128, May 1999.

[5] S. Bäro, G. Bauch, and A. Hansmann, "Improved codes for space-time trellis-coded modulation," IEEE Commun. Lett., vol. 4, pp. 20-23, Jan. 2000

[6] C. Loo, "A statistical model for a land mobile satellite link," IEEE Trans. Veh. Technol., vol. VT-34, pp. 122-127, Aug. 1985.

[7] M. Uysal and C. N. Georghiades, "Error performance analysis of space-time codes over Rayleigh fading channels," J. Commun. Networks, vol. 2, no. 4, pp. 351-355, Dec. 2000.

[8] G. Taricco and E. Biglieri, "Exact pairwise error probability of space-time codes," IEEE Trans. Inform. Theory, vol. 48, pp. 510-513, Feb. 2002.

[9] M. K. Simon, "Evaluation of average bit error probability for space-time coding based on a simpler exact evaluation of pairwise error probability," J. Commun. Networks, vol. 3, no. 3, pp. 257-264, Sept. 2001.

[10] M. K. Simon and M.-S. Alouini, Digital Communication Over Fading Channels: A Unified Approach to Performance Analysis. New York: Wiley, 2000.

[11] G. L. Turin, "The characteristic function of Hermetian quadratic forms in complex normal random variables," Biometrika, pp. 199-201, June 1960.

[12] A. M. Mathai and S. B. Provost, Quadratic Forms in Random Variables: Theory and Applications. New York: Dekker, 1992.

[13] J. Huang and L. L. Campbell, "Trellis coded MDPSK in correlated and shadowed Rician fading channels," IEEE Trans. Veh. Technol., vol. 40, pp. 786-797, Nov. 1991.

[14] R. G. McKay, P. J. McLane, and E. Biglieri, "Error bounds for trellis coded MPSK on a fading mobile satellite channel," IEEE Trans. Commun., vol. 39, pp. 1750-1761, Dec. 1991.

[15] M. Uysal and C. N. Georghiades, "Analysis of concatenated trellis coded STBC schemes over Rician fading channels," in Proc. 39th Annu. Allerton Conf. Communications, Control, Computing, Oct. 2001.

[16] I. S. Gradshteyn and I. M. Rzyhik, Table of Integrals, Series, and Products, 5th ed. San Diego, CA: Academic, 1994. 